\title{
Recovering Valuable Phosphates: Chemical Biotechnology as a Problem Solver for the Environment
}

Elsbeth Heinzelmann, science + technology journalist

Abstract: Researchers from the HES-SO Valais/Wallis have demonstrated how to extract phosphate from sewage sludge on the laboratory scale using renewable energy sources from a microbial fuel cell. The mobilized phosphate barely contains heavy metals and can be used to produce fertilizer of marketable quality. The necessary energy comes from a sewage treatment plant and causes no additional costs.

Keywords: Microbial fuel cell · Phosphate recovery · Phosphate remobilization - Sewage sludge $\cdot$ Struvite fertilizer

The shock was deeply felt in 1973: All of a sudden it dawned on us that our oil reserves were limited and that we were faced with considerable energy problems. But another global catastrophe is developing inevitably and entirely in secret, but hanging like the sword of Damocles above mankind: the shortage of phosphate. This charged particle (ion) contains the mineral phosphorus, which our body needs to build and repair bones and teeth, to support nerve function and muscle contraction. Nearly $85 \%$ of the phosphorus contained in the body occurs as phosphate in our bones, the rest is located in our tissues.

\section{How to Save a Precious Resource}

One dramatic aspect is that up to $80 \%$ of the deposits are held by just a few countries, Europe is dependent on imports for up to $90 \%$ of its supply. Phosphate is mined as a raw material for the most part in Morocco and Western Sahara, China, South Africa and the USA. Experts estimate that up to 160 million tons

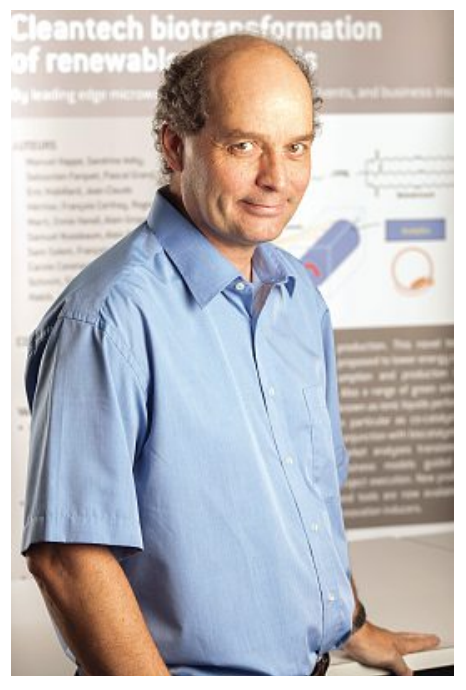

Professor Fabian Fischer, project leader and responsible for the Laboratory for Chemical Biotechnology at the HES-SO in Sion. Photo Denis Emery. a year is extracted worldwide. $80 \%$ goes into the processing of fertilizers, with potash and nitrogen constituting the most important nutrients in agriculture. But the end of an era is dangerously imminent: Phosphate is becoming increasingly scarce due to its deteriorating quality and the fact that it is becoming increasingly expensive to remove all impurities from this material. But in contrast to oil, there is no alternative to phosphate.

Probably the most important concentrated phosphate source for the future is phosphate contained in waste. A very promising method for phosphate recycling is offered by microbial fuel cells (MFC), as they combine effectively with wastewater treatment and nutrient extraction. In these cells, potassium cations migrate from the anode to the cathode. This cation migration allows sustainable chemical base generation, which remobilizes phosphate from insoluble iron phosphate contained in digested sewage sludge. "This approach is also useful for other industrial processes where a chemical base is needed", explains Fabian Fischer, Professor in Chemical Biotechnology at the HES-SO Valais/Wallis in Sion. In a study supported by the Swiss Federal Office for the Environment (FOEN) and industrial partners, he investigated how microbial electrolysis cells accelerate phosphate remobilization from iron phosphate contained in sewage sludge.

\section{Get to the Bottom of Things}

"Using microbial fuel cell (MFC) power we were able to extract up to $82 \%$, or $600 \mathrm{mg} \mathrm{L}^{-1}$, of the phosphate from digested sewage sludge", explains the chemist. "In a second step, we transformed the remobilized phosphate into what is known as struvite (magnesium ammonium phosphate), a phosphate mineral."

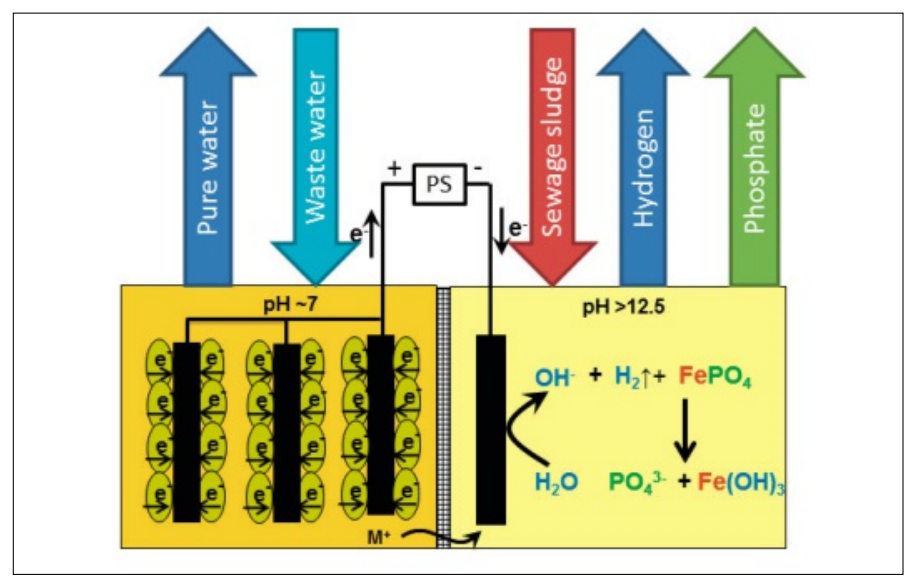

Production of phosphate from sewage sludge. Graphics by Institute of Life Technologies, Env. Sci. Process. Impact. 2015, 17, 90-97.

Based on the findings of Fabian Fischer and his team, other research groups also investigated the MFC approach. One used swine manure in a single-chambered MFC, where phosphate was deposited as struvite in the vicinity of an air cathode. Microbial electrolysis cell conditions also caused struvite deposition on hydrogen releasing cathodes. The researchers also noted combined nitrogen and phosphate recovery when using microbial fuel cell power. "Likewise, there are non-bioelectric processes such as electrodynamic recovery from packed beds and electrochemical phosphate isolation from nanofiltration concentrates", comments Fabian Fischer.

Although Fabian Fischer and his team discovered that microbial fuel cell-enabling phosphate remobilization from insoluble $\mathrm{FePO}_{4}$ was potentially quantitative, it was still very slow. In order to improve reaction rates, the scientists need to understand the remobilization mechanisms in order to scale-up the process. As earlier results have shown, electrochemical reduction occurs under microbial fuel cell conditions, as iron content increases 
in iron-phosphate particles. The research team also noted a $\mathrm{pH}$ increase up to $\sim 9$ in the abiotic cathode using a dual-chambered microbial fuel cell. "This is based on the fact that iron and oxygen are reduced in the cathode and a base $\left(\mathrm{OH}^{-}\right)$is formed, which stays in place because alkali cations $\mathrm{M}^{\mathrm{n}+}$ migrate into the cathode", says Fabian Fischer. "Therefore, a basic iron phosphate substitution mechanism is a second reaction mechanism". In the scope of this work, a supplemental voltage was applied to a dual-chambered microbial fuel cell to improve phosphate remobilization rates. This transforms the MFC into a microbial electrolysis cell. Particle-fluid kinetics was elaborated to identify the reaction resistance in the phosphate remobilization process.

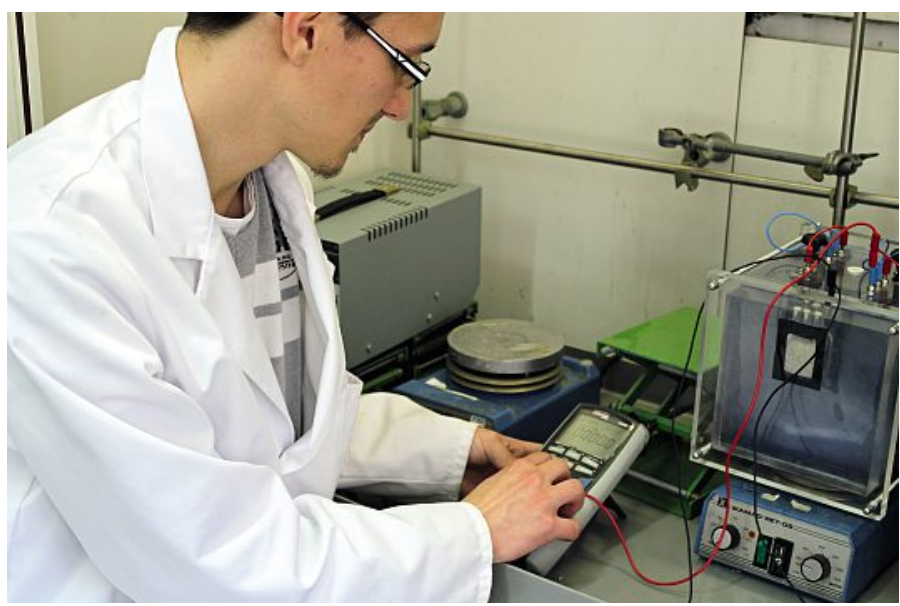

Maxime Blatter is making an experiment at the microbial fuel cell which can be used optionally as a microbial electrolytic cell. $\mathrm{He}$ is an EPFL student who completes his master studies at the Institute of Life Technologies in Sion. Photo Elsbeth Heinzelmann.

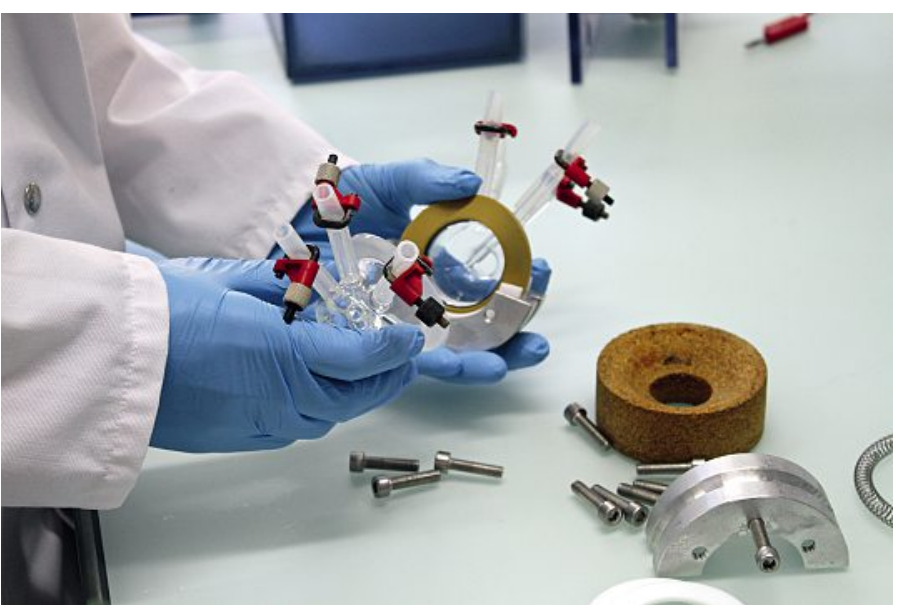

The microbial fuel cell made of glass serves in this project for experiments under sterile conditions. Photo Elsbeth Heinzelmann.

\section{New Scientific Findings Pave the Way for the Future}

For the scale-up process of phosphate remobilization, two three-litre MFC reactors and a twelve-litre MFC reactor were built. The examination of the process management revealed that the microbial electrolysis mode delivered the best results from all parameters. It was possible to produce a quantitative yield $(95 \%)$ significantly faster. "In the three-litre scale-up reactor we managed to achieve a remobilization of $67 \%$. As kinetic analyses show, extremely high values of up to $98 \%$ are possible". An important finding is the proof of reaction mechanisms. "As a result of this examination, the reductive mechanism is only important when slow remobilization takes place."

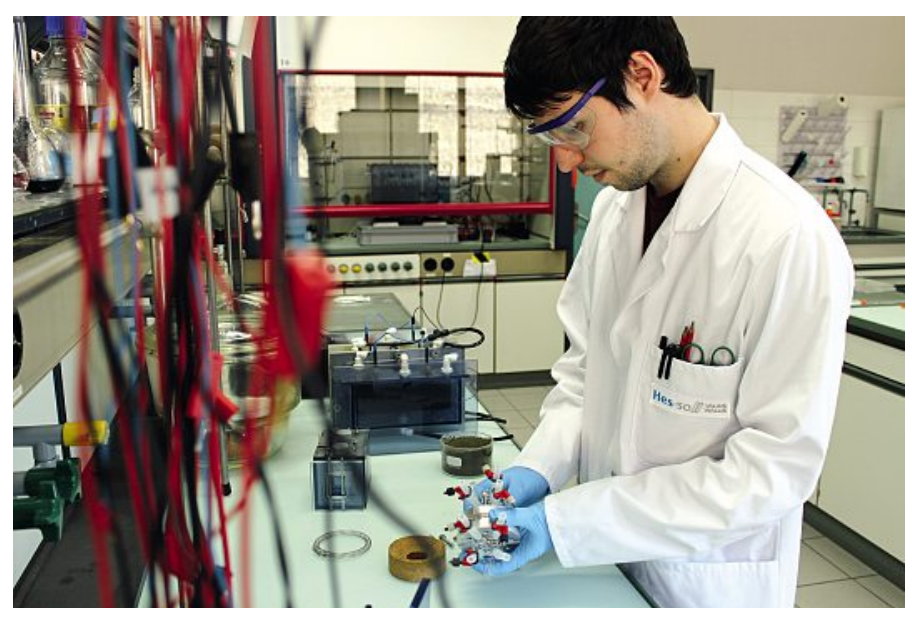

Marc Sugnaux, scientific collaborator at the Institute of Life Technologies, prepares a microbial fuel cell. Photo Elsbeth Heinzelmann.

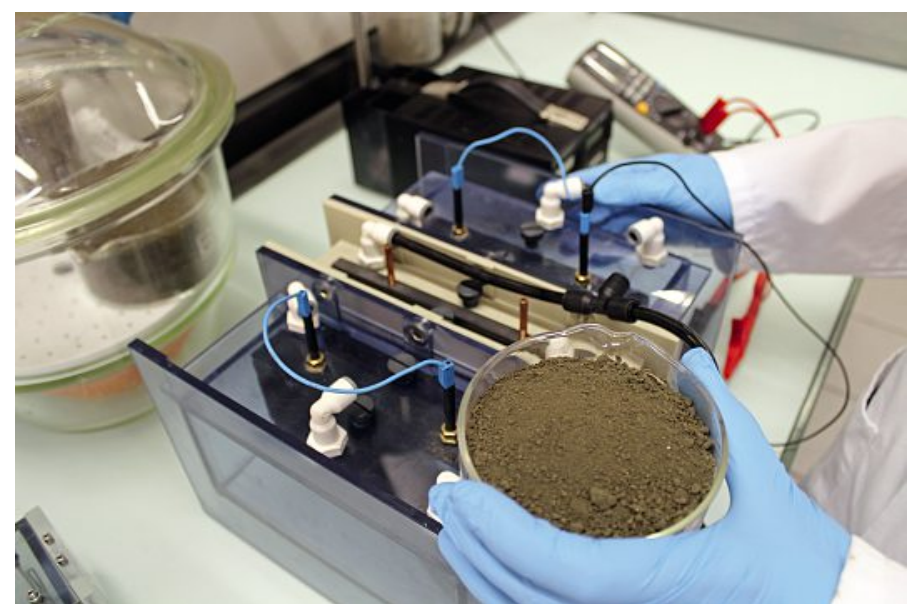

Marc Sugnaux shows de-watered and digested sewage sludge prior to phosphate extraction in the microbial fuel cell. In the middle of the reactor, the abiotic cathode for phosphate remobilization, to the left, the bio-anode in the wastewater stream for the production of bio electricity. Photo Elsbeth Heinzelmann.

An important influence is exerted by the predominant $\mathrm{pH}$ level. This level allows a fast substitution mechanism and is particularly virulent under electrolysis conditions. Apart from the strong $\mathrm{pH}$ dependence, the researchers observed a temperature effect, with an acceleration of the process at a slightly elevated temperature. Fabian Fischer's group analysed the produced struvite fertilizer by inductively coupled plasma mass spectrometry (ICP-MS). The investigation confirmed that the levels of cadmium, lead and other heavy metals in recycled fertilizer could easily be kept below the legal limits.

On the one hand, the new remobilization process allows all the phosphorous to be recovered from sewage sludge. This creates the basis for the production of phosphate-free sewage sludge for incineration in solid waste incineration plants or for cement manufacture. "It is quite conceivable that the chemical base can be used for other purposes, for instance the neutralization of acid industrial wastewater", predicts Fabian Fischer. He now intends to build a larger reactor. The investigation project consortium will continue and be supplemented by further industrial partners. "For the time being, we are planning a pilot plant to produce higher quantities of fertilizer in order to acquire more experience with the system."

Further Information: fabian.fischer@hevs.ch

URL: www.hevs.chw 